TM-1452

(SSC-N-332)

0102.000

\title{
A One-Campus SSC
}

\author{
L.M. Lederman and L.C. Teng \\ Fermi National Accelerator Laboratory \\ P.O. Box 500, Batavia, Illinois 60510
}

April 1987 
TM-1452

April, 1987

\section{A One-Campus SSC}

L. M. Lederman and L. C. Teng

Fermi National Accelerator Laboratory

Batavia, Illinois 60510

\section{Introduction}

We extend the argument made briefly in SNOWMASS $186^{1}$ that a bypass-clustered IR arrangement could simplify the operational problems and save considerable money. The basic idea is to have only one campus containing all IR's, distributed on bypasses, and the injector machines. It should be emphasized that the bypass-clustered IR geometry is an essential part of the single-campus concept. It is not realistic or efficient to consider a long narrow campus when all six or so IR's are strung out in one line. The single-campus bypass-clustered IR arrangement has the following virtues:

The major $4 \pi$ detectors can be built in-place and each serviced during the (say) five months of the year that the beams are servicing the detectors on another bypass. Smaller and relatively easily removed detectors can still be alternated in a push-pull arrangement in the unbypassed utility straight sections.

The efficiency of operation for both the collider and the experimental detector facilities is greatly improved. This is especially true for the operation and maintenance personnel of the collider.

The need for support facilities: shops, computer centers, libraries, cafeteria, stockrooms, lecture halls are cut almost in half since all of these would have to be duplicated in a two-campus 
arrangement. This also applies to utility distributions: water, electricity, waste disposal.

(4) The communications both with reference to scheduling, and maintenance and the intellectual stimulation of seminars, workshops, etc. are vastly improved. Another vital element is the availability of the engineering staff, purchasing, personnel services and the much greater ease of DOE supervision (sic!).

(5) The manpower levels of support staff are lower since one loses less in staffing for peak loads.

(6) Test beams are now conveniently available to all users rather than just $30 \%$ of them as in the conceptual design.

(7) Land acquisition is halved and whereas this may only affect state funds, there are inevitable hostilities that are derived from land acquisition. Also taxpayers are involved even with state funds. Alternatively, the "other" campus can be reserved for future expansion.

(8) Concentrating activities in a single campus also simplifies safety, fire protection, security.

(9) We will need fewer roads and fewer buildings.

(10) Finally and perhaps most importantly, this arrangement makes it very practical to bend the machine so that the interaction regions are as close to the surface as shielding requirements permit. ${ }^{2}$

Given all these virtues we examine below various possible geometries and arrangements, adopted from various CDG publications. 
II. Possible arrangements

If no more than six IR's are desired, two bypasses are sufficient. A double bypass is simplest because the two branches can be totally symmetric. It is even possible to have eight IP's by adding "simple" IR's symmetrically in the main trunk. Injection has to be into the main trunk-line. Thus we arrive at the 4 arrangements shown in Fig. 1.

Case a. There are six IP's, three on each bypass. Injection is into two neighboring utility straights on the trunk line at one end of the bypass. If the campus is to cover all entrances to the straight sections it should extend from the midpoint of the first straight section to that of the last straight section. The length of the campus is therefore

$$
4\left(\mathrm{~L}_{\mathrm{s}}+\mathrm{L}_{\mathrm{c}}\right)=9.6 \mathrm{~km}
$$

where

$$
\begin{aligned}
& \mathrm{L}_{\mathrm{s}}=\text { length of straight section }=1.152 \mathrm{~km} \\
& \mathrm{~L}_{c}=\text { length of curved section }=1.248 \mathrm{~km}
\end{aligned}
$$

Since the high energy booster (HEB) has a diameter of $\sim 2 \mathrm{~km}$, the width of the campus should be $\tilde{>} 3 \mathrm{~km}$ in all cases.

Case b. There are four IP's two on each bypass. Injection is identical to case a. The necessary length of the campus is

$$
3\left(\mathrm{~L}_{\mathrm{s}}+\mathrm{L}_{\mathrm{c}}\right)=7.2 \mathrm{~km}
$$

Case c. Access to the utility straights may not have to be contained inside the campus area. It is then advantageous to locate the two injection utility straights symmetrically at both ends of the double bypass. For six IP's the arrangement looks like that shown and the necessary campus length is

$$
2\left(L_{s}+L_{c}\right)=4.8 \mathrm{~km}
$$


The injection beam lines are longer in Case c. But as has been shown from Fermilab experiences these beam lines can be very simple and inexpensive. If the HEB is bipolar and the oppositely circulating beams are extracted from the same circumferential location it is more convenient to inject into the two SSC rings from the same side. If the HEP is unipolar it is more advantageous to extract from two different circumferential locations for injection into the two SSC rings. Injection can then be from the same side or from opposite sides if the HEB straddles the SSC tunnel as shown in the diagram.

Case d. With one utility straight at each end, the four IP arrangement looks like that shown in this case and the necessary campus length is only

$$
\left(\mathrm{L}_{\mathrm{s}}+\mathrm{L}_{\mathrm{c}}\right)=2.4 \mathrm{~km}
$$

For this arrangement a squarish campus of $\sim 3 \mathrm{~km} \times 3 \mathrm{~km}$ (about half that of Fermilab) is adequate. Efficient operation within such a single campus is well borne out by Fermilab experiences.

III. Technical Details

Most of the designs of lattice components can be taken directly from the Conceptual Design Report.

1. The designs of the Interaction Region Module (Interaction Straight), the Utility Module (Utility Straight) and the 106 milliradian curved section joining these zero-dispersion straights are as given in the CDR.

2. For Cases b and $d$ a half-curved section of 53 milliradian which matches an Interaction Straight to an Utility Straight must be designed. The length of this half-curved section is flexible, but will presumably be 
not too different from $L_{c} / 2=624 \mathrm{~m}$. The design of such a section is straightforward and is not expected to encounter difficulties.

3. The switching between the two bypass branches is best carried out in the central $660 \mathrm{~m}$ long drift spaces of the utility straights at the ends of the bypass. This is shown in Fig. 2. The switching dipole M is located at the upstream end of the drift space. The minimum orbit separation at the downstream quadrupole $Q$ is $\sim 0.6 \mathrm{~m}$. The orbit deflection by the switching magnet must therefore be $\star 0.3 / 660=\star 0.5$ mrad. Since the bend angle of each ring dipole is $\sim 1.6 \mathrm{mrad}$, the switching magnet strength is only less than $\sim 1 / 3$ that of a ring dipole. The small bend angle and dispersion imported to the orbit by the switching dipole can easily be compensated by slight readjustments of a few quadrupoles and dipoles downstream of the central drift space.

IV. Conclusion

The one-campus SSC with bypass-clustered IR arrangement has many virtues and can be easily designed. Designs for double-bypass arrangements with 4 and 6 IP's are examined and presented here. Compared to the Conceptual Design given in the CDR, the only drawback is the additional dipoles required which amounts to $\sim 20 \%$ for the 6 IP arrangements and $\sim 10 \%$ for the 4 IP arrangements. On the other hand, we have assumed the same 106 mrad curved sections between Interaction Straights for isolation of events from neighboring IP's as in the CDR. If a smaller angle is sufficient for isolation there is a consequent saving. 


\section{Figure Captions}

Figure 1. Four different arrangements for a double-bypass. Cases a and $c$ each have six IP's and Cases $b$ and $d$ each have four IP's. (not to scale) Additional IP's may be inserted into the trunk lines if needed.

Figure 2. Scheme for switching between bypass branches. (not to scale)

\section{References}

1. SNOWMASS ' 86 to be published.

2. See for example: Three Dimensional Ring Configuration, SSC Central Design Group, April '86. 


\section{Case a}

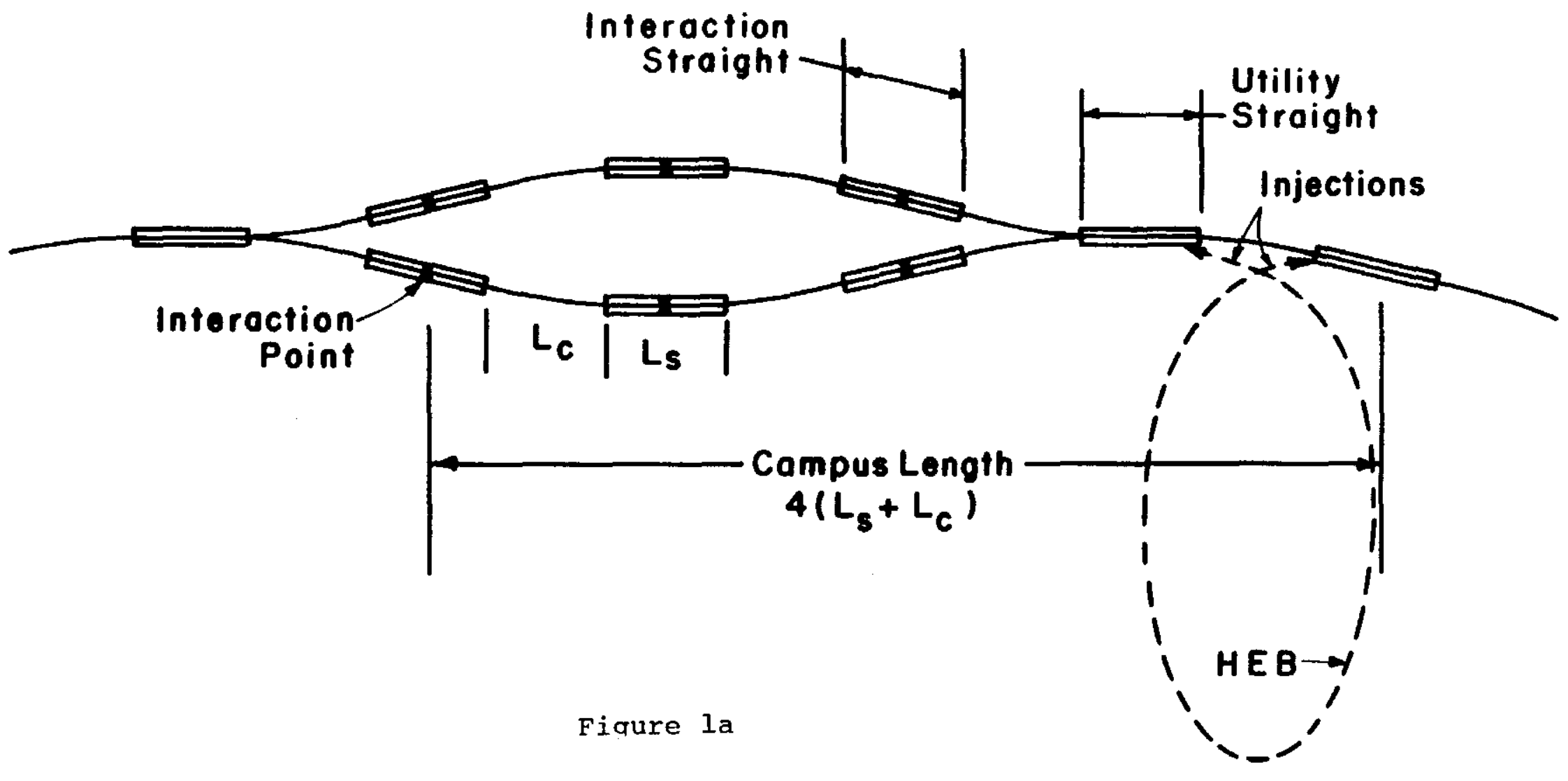




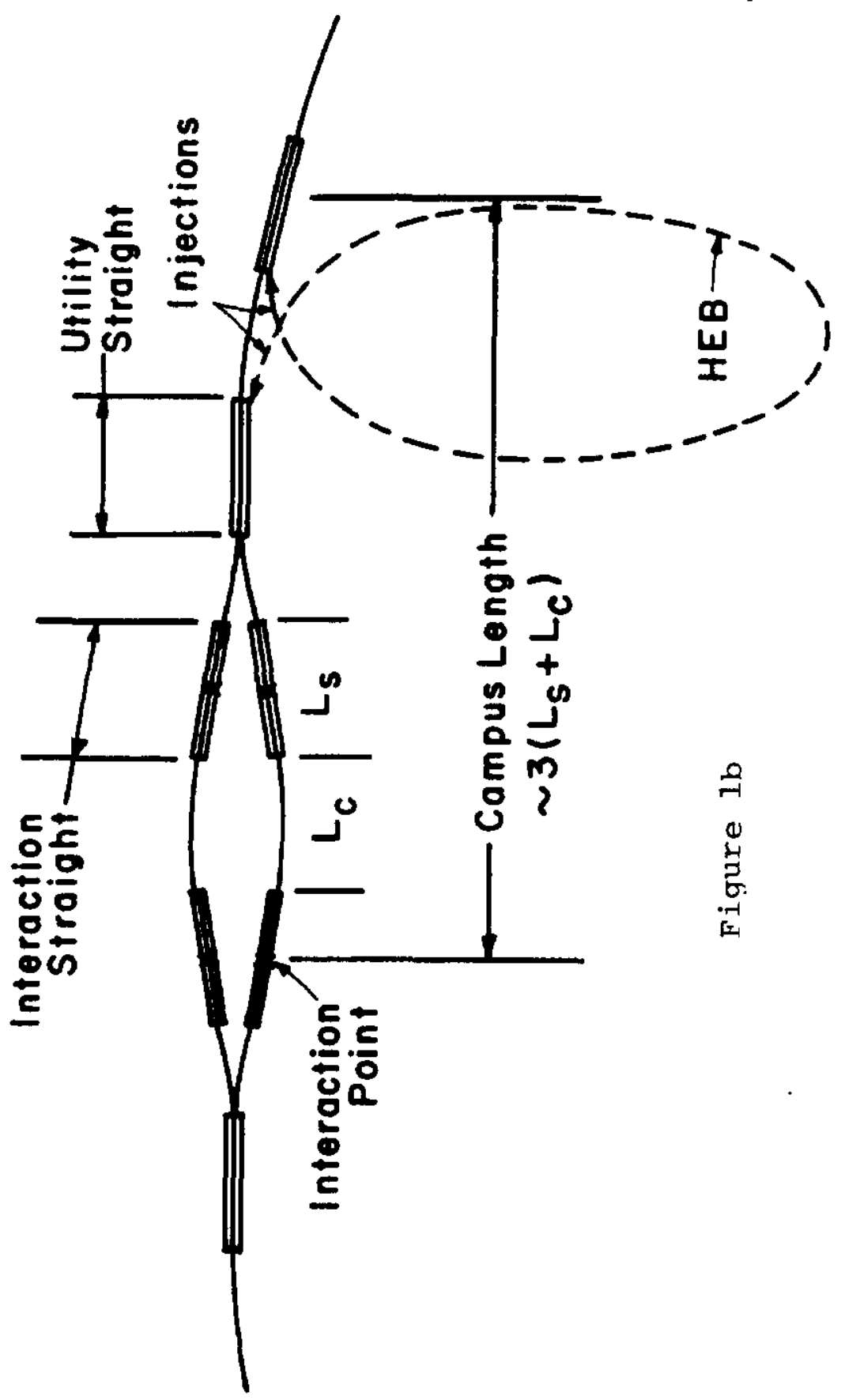

0
0
0 


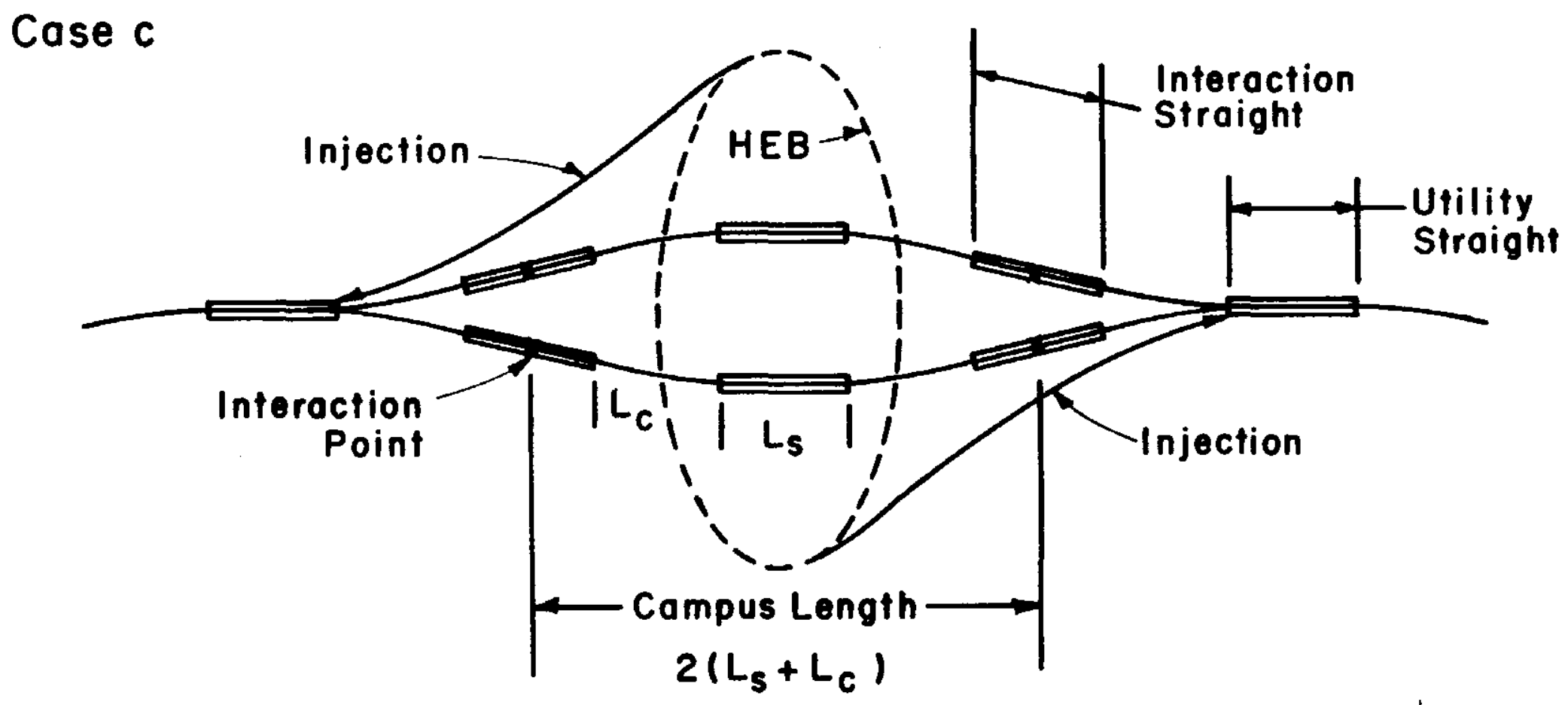

Fiqure lc 


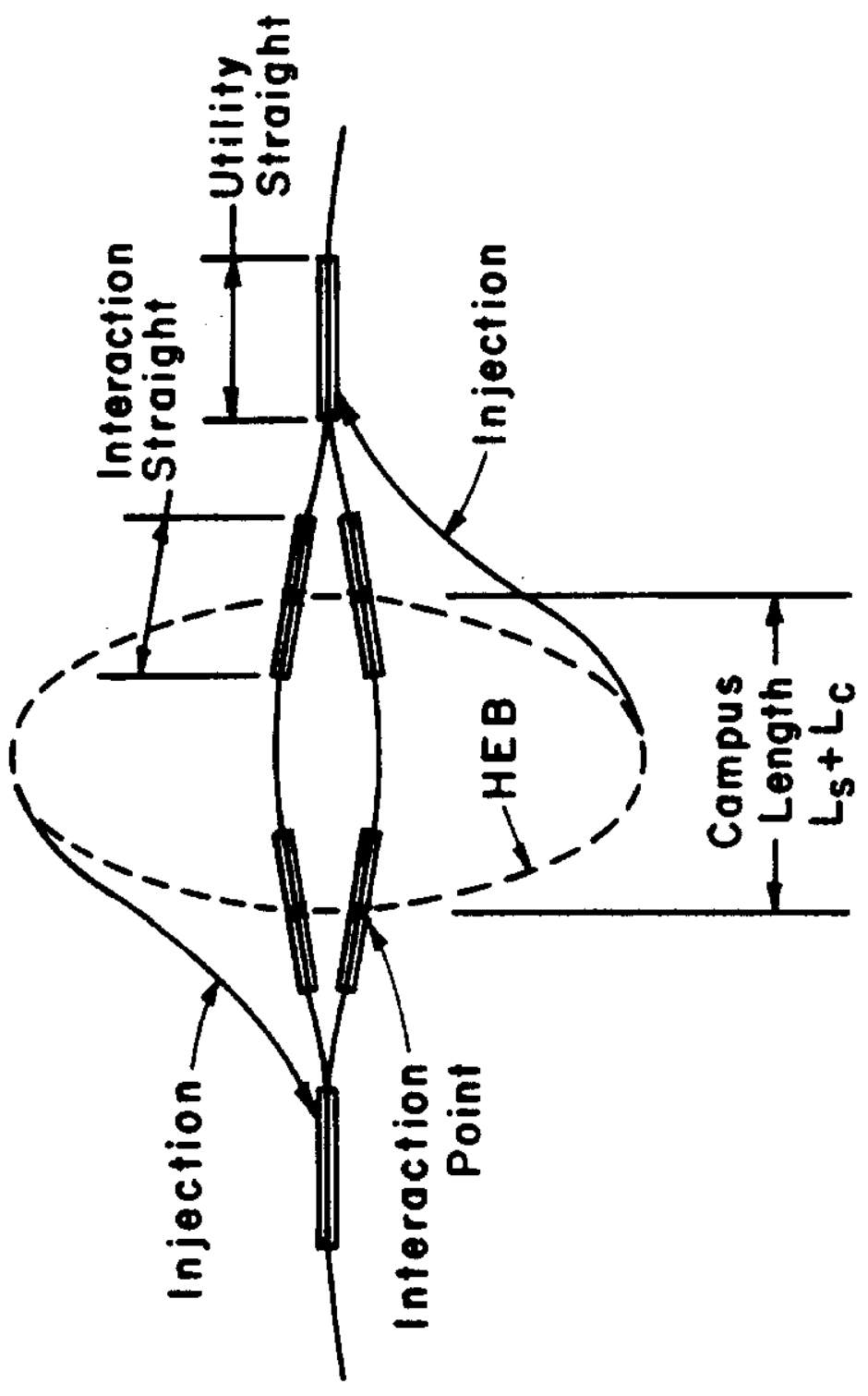

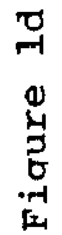

0
0
0
0 


$$
y
$$

